\title{
SAÚDE INTERNACIONAL: UMA DAS POSSIBILIDADES DE ATUAÇÃO DA ENFERMAGEM NO CAMPO DA SAÚDE COLETIVA
}

Maria Rita Bertolozzi*

BERTOLOZZI, M.R. Saúde internacional: uma das possibilidades de atuação enfermagem no campo da saúde coletiva. Rev.latino-am.enfermagem. Ribeirão Preto, v. 4, n. 1, p. 87-95, janeiro 1996.

Presente artigo relata a experiência de participação em um programa no campo da Saúde Internacional, que vem sendo promovido pela Organização Panamericana da Saúde. A partir da síntese do conceito de Saúde Internacional, a autora descreve os objetivos do Programa bem como as atividades desenvolvidas para a consecução dos mesmos. Após essa exposição aponta-se a necessidade de que a categoria de Enfermeiras participe do mesmo, no sentido de ampliar a visibilidade do saber e do fazer em Enfermagem.

UNITERMOS: saúde internacional, cooperação técnica em saúde

\section{CONSIDERAÇÕES INICIAIS}

O presente artigo apresenta um relato de experiências junto ao Programa de Formação em Saúde Internacional, promovido anualmente pela Organização Panamericana da Saúde, em Washington, DC-EUA.

*Professor-Assistente do Departamento de Enfermagem em Saúde Coletiva da Escola de Enfermagem da Universidade de São Paulo 
A difusão internacional das enfermidades transmissíveis, as importantes transformações do perfil epidemiológico de diversas formações sociais, além das tentativas de investigadores em expandir a estrutura explicativa do processo saúde como uma área significativa no interior do campo das relações internacionais concebido como uma superestrutura política, econômica e militar que regula a relação entre as nações - vêm orientando a conceptualização da saúde como um tema de ordem internacional.

A Saúde Internacional é um campo de estudos e de prática profissional que considera que o setor saúde não se encontra à margem das grandes tendências mundiais: de dependência entre os países e da globalização dos mercados. O desenvolvimento econômico e a saúde vêm se tornando intimamente interrelacionados, o que vêm compelindo alguns governos à revisão do papel do Estado no que toca à regulação da provisão de alguns bens e direitos. Como resultado disso, cada vez mais, uma série de organizações internacionais tem concebido a saúde como uma questão preponderante para a paz e para a maior solidariedade entre as nações.

Muitos são os trabalhos (UNDP, 1990, TERRIS, 1988; SEAH, 1976; BAKER et al. 1984; GIOVANNINI, 1982) que têm evidenciado interesse no aprofundamento do conhecimento das diversas formas de abordagem da questão da saúde no âmbito internacional e que tratam dos determinantes dos complexos problemas de saúde dos países da região, para criar mecanismos efetivos de intervenção para a sua transformação. A complexidade do tema "Saúde Internacional" induz à busca de alternativas de instrumentos, de métodos de análise, além de um pensar que vá além dos tradicionalmente aplicados para estudar o processo saúde-doença.

De modo geral, as questões relativas à problemática da saúde em nível internacional têm sido pouco incorporadas aos processos formativos do pessoal de saúde, seja da área de serviços, de educação ou de investigação, nem tampouco têm elas se constituído em preocupação da maioria do corpo docente responsável por programas de pós-graduação na área de Saúde Coletiva.

O Human Resources Development Program, da Pan-American Health Organization (PAHO), especialmente nos últimos 20 anos, vem apoiando os esforços regionais no desenvolvimento da força de trabalho no campo da Saúde Pública e o desenvolvimento da Medicina Social na América Latina, evidenciando experiências que vem sendo levadas a cabo com o intuito de fortalecer o potencial da PAHO/WHO (World Health Organization).

Assim sendo, a prática na PAHO, organizada em conseqüências de distintas e, muitas vezes contraditórias teorias e concepções ideológicas, oferece a possibilidade de se entender a magnitude dos determinantes internacionais sobre a saúde, na medida em que integra, no seu dia-a-dia, a intervenção de distintos atores sociais que atuam diretamente no amplo domínio da Saúde Internacional.

Desde 1985, a Organização Mundial da Saúde aponta a necessidade do desenvolvimento de lideranças na saúde como uma das estratégias para a transformação da situação de saúde. A região das Américas, na tentativa de 
responder a esse compromisso vem promovendo o Programa de Formação em Saúde Internacional (também conhecido como residência em Saúde Internacional), através do seu Programa Especial de Desenvolvimento de Recursos Humanos da Organização Panamericana da Saúde, na oficina central, em Washington DC, USA.

Portanto, este presente artigo objetiva relatar a experiência de participação em um Programa no campo da Saúde Internacional.

O profissional que é selecionado para participar do Internacional Health Program deve ter experiências prévias na pós-graduação em Saúde Pública e na prática de assistência, docência ou investigação em saúde. Esses pré-requisitos favorecem a sua incorporação no processo reflexivo e analítico de estudo-trabalho sobre a Cooperação Técnica que realiza a PAHO na Região das Américas, tanto no que toca às suas características principais enquanto organismo intergovenamental de Cooperação Internacional, como de sua interação com outros organismos de Cooperação.Ao mesmo tempo, o processo de estudo-trabalho no interior da PAHO permite o aprofundamento no campo de especialidade do profissional selecionado, bem como na participação do desenvolvimento de projetos de cooperação em nível internacional.

Nesse sentido, as práticas de Cooperação Técnica da PAHO se constituem no eixo em que se articula o Programa, no interior do qual, durante 11 meses de trabalho, é possível conviver, interatuar, criticar e analisar o processo onde se encontram incorporadas a formulação de políticas, a análise e formulação de estratégias, planos e programas, além da discussão sobre os recursos financeiros, até o momento de sua aprovação em nível dos corpos diretivos da Organização.

Após um período inicial onde são fornecidas orientações básicas para a integração no Programa, se sucede outro, que consiste fundamentalmente na participação em seminários, em reuniões de grupos técnicos, comitês e corpos consultivos, diretivos e executivos da Organização, onde é possível, paulatinamente, adquirir conhecimentos sobre a estrutura e função na Organização, bem como sobre seu desenvolvimento histórico e sua interação com agências de cooperação. Cabe dizer que o aprofundamento no processo de Cooperação Técnica com os países requer conhecimento e interiorização sobre as condições de vida e de saúde das populações, de seus determinantes sociais, das concepções políticas, assim como das formas de abordagem dessas questões em nível nacional e internacional e, para tanto, o Programa contempla no seu decorrer, momentos de reflexão e estudo sobre estas questões.

\section{OBJETIVOS DO PROGRAMA}

Casa um dos objetivos do Programa de Formação em Saúde Internacional será apresentado de modo articulado às atividades desenvolvidas a fim de possibilitar 
uma visão mais ampla da essência do seu desenvolvimento.

Primeiro objetivo: "Aprofundar no conhecimento da situação de saúde dos países da região das Américas e de seus determinantes políticos, econômicos, sociais e conjunturais, em nível local, sub-regional, regional e internacional e aprofundar na abordagem dos problemas fundamentais de saúde da Região, além das alternativas de transformação dos sistemas de saúde frente ao horizonte de Saúde para Todos".

Para cumprir esse objetivo foram programadas visitas a alguns países: Chile, México, Argentina, Brasil, Canadá e para algumas regiões dos Estados Unidos da América, o que permitiu conhecer a complexa situação de vida e de saúde desses locais, além de evidenciar as imensas contradições em termos de realidade de vida entre os grupos sociais que neles vivem. Além disso, ressaltamse os elementos proporcionados pelas leituras realizadas, pela socialização do conhecimento nas discussões em grupo com os demais profissionais participantes do Programa, os quais durante o ano de 1993 eram provenientes do Chile, Costa Rica, Equador, Guatemala, Nicarágua, Peru e Colômbia, bem como através do contato com especialistas em diversas áreas do conhecimento, oriundos de distintos paises. A participação nas reuniões dos Conselhos: Diretivo, Executivo e em outros fóruns consolidou uma visualização ampla da problemática que envolve a região das Américas.

Cabe ressaltar que a participação nas discussões com os Ministros de Saúde de todos os países das Américas, além da visita a alguns serviços de saúde dos países anteriormente citados, como ambulatórios, centros comunitários, centros de atenção à saúde dos imigrantes, hospitais, bem como a visita a Universidades e às sedes das Representações da PAHO nesses paises, igualmente promoveu a consecução do objetivo proposto.

O segundo objetivo era: "Conhecer as características da PAHO frente à problemática de saúde dos países, tomando as experiências dos mesmos como base para a análise da Cooperação Técnica em saúde". Para atingir esse objetivo foi programado em ciclo de seminários que tinha por finalidade a discussão sobre os conceitos de Saúde Internacional, Cooperação Técnica e a Planificação Estratégica como um método de abordagem da Saúde Internacional. Com isso, foi possível explorar os objetos de estudo da Saúde Internacional e da Cooperação Técnica, além de tomar os primeiros contatos com as práticas de Cooperação Técnica entre os países.

As visitas realizadas aos países já mencionados, as leituras de vários documentos sobre os temas em questão, a experiência que o trabalho cotidiano no Human Resources Development Special Program (Programa onde a autora concentrou o desenvolvimento de suas atividades) ao lado da participação em reuniões dos Conselhos e com os profissionais Representantes da PAHO em cada um dos países das Américas, foram elementos-chave para a consecução desse segundo objetivo. 
O terceiro objetivo era: "Desenvolver a capacidade para a análise, formulação e avaliação de programas de Cooperação Técnica em saúde". Foi através da participação no trabalho realizado nas diversas instâncias de deliberação na PAHO - cujas atividades se encontram detalhadas no documento a seguir - e no curso de capacitação "Logical Framework" - que desenvolve habilidades na formulação e avaliação de projetos que são encaminhados a organizações internacionais na busca de financiamento - que se buscou alcançar o objetivo proposto. A oportunidade de discutir o conteúdo de vários projetos em diferentes etapas de desenvolvimento enriqueceu o desenrolar do Programa, principalmente pela possibilidade de verificar numa dimensão muito mais complexa e ampla as questões relativas às necessidades de saúde dos grupos populacionais nas Américas.

O quarto objetivo era: "Conhecer as políticas de saúde adotadas pelos países no plano da Cooperação Internacional, suas metas e implicações na organização do setor saúde". Além das atividades programadas oficialmente no interior do Programa, é necessário ressaltar que foi possível acompanhar o processo de negociações para a reforma do Sistema de Saúde dos Estados Unidos da América Latina. Essas questões se referem ao papel do Estado como regulador do setor, à tendência à privatização dos serviços de saúde, além de outras referentes à imposição da tecnologia na medicina curativa e individualista em contraposição à prevenção e promoção à saúde, à simplificação e eficiência da assistência, ao direto à saúde e ao papel das negociações de caráter "lobbista" na elaboração das políticas públicas.

É necessário explicitar que foi possível visualizar, de forma bastante ampla, várias experiências de Cooperação Internacional entre os países, dentre as quais ressaltam-se os projetos entre a Nicarágua e Estados Unidos na área de Enfermagem, ou entre o primeiro país citado e Cuba, no que toca à epidemia de neurite óptica que ocorreu neste país durante o primeiro semestre de 1993. Nesse sentido, é fundamental evidenciar a importância que tem o Brasil no campo da saúde, que apesar da grave crise social e econômica que vem se dando nos últimos anos, vem assumindo uma posição de vanguarda em relação às experiências nesse campo, no que toca ao papel da população e de grupos organizados na evidenciação do resgate da democratização e da honestidade do setor público, como pode ser visto através do estudo sobre o processo de reforma do Sistema de Saúde do nosso país. Essas experiências, de fato, necessitam ser difundida, na medida em que constituem elementos básicos que podem subsidiar a discussão sobre a estruturação dos Sistemas de Saúde em países da América Latina e Estados Unidos. Por outro lado, ficou bastante claro que os países da Américas também muito podem oferecer em termos de Cooperação Técnica para o Brasil como, por exemplo, a experiência canadense e estruturação do Sistema de Saúde, ou mesmo as experiências de estruturação curricular na qualificação da força de trabalho da 
saúde, como no caso da Universidade Autônoma de Xochimilco, no México.

Os objetivos cinco e seis eram, respectivamente: "Conhecer os diferentes organismos que interatuam ou intervém, técnica ou financeiramente no desenvolvimento do setor saúde nos paises do Continente" e "Desenvolver a capacidade para mobilização de recursos, formulação, execução e avaliação de projetos". Para cumprir esses objetivos foram planejadas visitas e reuniões de discussão em organismos financeiros, como a Canadian International Development Agency (CIDA), a United Nations Fund Population Assistance (UNFPA), o World Bank, o International Development Bank, entre outras agências, ocasião em que foi possível verificar o processo de desenvolvimento de projetos, desde a sua elaboração até os processos de negociação, aprovação e avaliação. Essas oportunidades enriqueceram o universo de conhecimento a respeito dos canais de financiamento de projetos para os países em desenvolvimento e incorporaram elementos fundamentais para o pensar em novos caminhos para trabalhar com as questões de saúde no Brasil e, mais especificamente, no interior das práticas de investigação nas escolas de Enfermagem.

O sétimo objetivo era: "Organizar e revisar criticamente o conhecimento científico-técnico referido à área de concentração" e o oitavo: "Analisar os programas de apoio em nível regional e internacional e colaborar na formulação e elaboração de estudos, alternativas ou projetos que contribuam à solução dos problemas identificados no objetivo de número sete". Conforme me apontado anteriormente, uma vez que as atividades da autora se concentraram junto ao Human Resources Development Special Program, foi possível:

- participar de projetos que vêm promovendo a capacitação avançada de profissionais responsáveis pelo desenvolvimento da força de trabalho em saúde na região das Américas (e, nesse campo se incluem os componentes relativos à informação, investigação e planificação estratégica da força de trabalho em saúde);

- participar de projetos que vêm desenvolvendo a metodologia da educação permanente e que vêm sendo aplicada no interior na Panamerican Health Organization, para a capacitação de pessoal no interior dos serviços de saúde (e, nesse campo, se incluem também a série de projetos de produção e disseminação de livros de texto);

- participar de projetos que vêm apoiando a maior participação da Universidade no processo de transformação das práticas de saúde (mobilizando as associações responsáveis pelas instituições educativas e apoiando o desenvolvimento da formação de profissionais, prioritariamente em Enfermagem e Medicina). É importante ressaltar que a participação na Reunião sobre "Las Perspectivas del Papel de la Universidad Latinoamericana em la Salud de la Población" trouxe à tona uma série de elementos chaves para a compreensão do papel da Universidade, como lócus fertilizador do conhecimento em saúde para a América Latina. Através do conhecimento sobre a situação do ensino universitário em países como México, Argentina, El Salvador, Colômbia, bem como do papel da UNESCO, da União 
Interamericana de Universidades, e das agências financeiras como o International Development Bank e o World Bank, foi possível compreender a complexidade que envolve a formação da força de trabalho em saúde, e da necessidade de atrelamento deste setor ao de políticas públicas, no sentido de transformar a qualidade do ensino e da assistência à saúde das populações.

- participar na promoção dos projetos que vêm desenvolvendo a temática sobre a Teoria e a Prática da Saúde Pública, estimulando a revisão de programas educativos e de planos de investigação neste campo.

No interior do Human Resources Development Special Program foi possível conhecer a dinâmica interna e externa de seu trabalho, com especial ênfase no campo da Educação em Enfermagem, e na articulação do Programa em outras Divisões da PAHO. No campo da Educação em Enfermagem é necessário mencionar que houve oportunidade de verificar a forma como os países solicitam Cooperação Técnica e, como a PAHO responde a essas solicitações, elaborando projetos, em alguns casos, ou auxiliando na sua elaboração e execução, em outros. Além disso, foi oportuna a aproximação à situação da assistência e do ensino de Enfermagem nos vários países da região, bem como verificar as necessidades de Cooperação Técnica neste campo.

O objetivo nove era: "Fomentar a comunicação e o apoio mútuo entre os

trabalhadores de saúde do continente no contexto da contribuição à paz e solidariedade entre os povos". A oportunidade de captar a situação social e de saúde dos países das Américas e de refletir sobre elas a partir da ótica de um organismo de caráter intergovernamental foi fundamental para ampliar a compreensão da globalização dos problemas de saúde. Com isso, foi privilegiada a análise a partir da dimensão estrutural, articulada às demais dimensões de intervenção na realidade: no interior dos grupos sociais e em nível individual, vislumbrando-se que as mais sérias questões de saúde podem ser solucionadas por intervenções junto ao coletivo oriundas de proposições conjuntas em nível regional.

\section{COMENTÁRIOS FINAIS}

Substancialmente, a participação no Internacional Health Program contribui, efetivamente, para reconhecer a necessidade de se conhecer e estudar a temática da Saúde Internacional quando se pretende atuar na assistência junto ao coletivo ou junto ao processo de qualificação da força de trabalho e no campo da investigação em Saúde Coletiva.

A riqueza oportunidades que foram oferecidas durante o desenvolver do Programa, se constituíram em momentos de aprendizado e de reflexão sobre a 
necessidade de transformação da realidade de saúde de muitos países da região das Américas. Essa é uma necessidade já apontada há muito, mas que cotidianamente se agudiza e nos remete à responsabilidade de a ela responder com urgência e compromisso social, enquanto instituições responsáveis pela qualificação da força de trabalho em saúde.

Finalizando, é preciso ressaltar que a diversidade de oportunidade do International Health Program deve ser compartilhada por outras profissionais na área de Enfermagem. Em 9 anos de duração do Programa, somente 5\% pertenciam à categoria de Enfermeiras que foram selecionadas para participar do mesmo. O processo de se viver o cotidiano em outro país, de idioma diferente, interagindo com grupos de profissionais, educadores, investigadores, com formação nos mais distintos campos de conhecimento em saúde, com variados níveis de graduação acadêmica, oriundos de diversos países, que compartilham de uma forma distinta de organização do processo de trabalho é extremamente desafiante, carregado de riscos, mas que por outro lado, fortalece o desenvolvimento profissional.

\section{INTERNATIONAL HEALTH: ANOTHER POSSIBILITY TO NURSES ACTION IN THE FIELD OF COLLECTIVE HEALTH}

This article presents a report about the participation in one program in the field of International Health witch has been promoted by Panamerican Health Organization. The author presents a synthesis of the concept of International Health, describing the objectives of the Program and the activities developed during the process to achieve it. Beside this, the author indicates the importance of the participation of nurses in this kind of Program in order to enlarge the visibility of nursing knowledge.

UNITERMS: international health, technical cooperation in health

\section{SALUD INTERNACIONAL: UNA DE LAS POSIBILIDADES DE ACTUACIÓN DE LA ENFERMERÍA EN EL CAMPO DE LA SALUD COLECTIVA}

Este artículo presenta un relato sobre la participación en un programa en el campo de la Salud Internacional, el cual ha sido promovido por la Organización Panamericana de la Salud. La autora hace una síntesis del concepto de la Salud 
Internacional, describe los objetivos del Programa las actividades que fueron desarrolladas durante el mismo. Además, enfatiza la importancia de la participación de las enfermeras en este Programa con el sentido de ampliar la visibilidad del saber y de qué hacer en Enfermería.

UNITERMOS: salud internacional, cooperación técnica en salud

\section{REFERÊNCIAS BIBLIOGRÁFICAS}

01. BAKER, C. et al. United States health professionals in international health work. Am.J.Pub.Health, v. 74, n. 5, p. 438-41, 1984.

02. FERREIRA, J.R. et al. Overview of the Quebec Meeting. In: PANAMERICAN HEALTH ORGANIZATION. International health: a north-south debate. Washington DC: Panamerican Health Organization. p. XVII-XXXIII, 1992.

03. GIOVANNINI, M. J.; BROWNLEE, A. T. The contribution of social science to international health training. Soc.Sci.Med., v. 16, p. 957-64, 1982.

04. ORGANIZACIÓN PANAMERICANA DE LA SALUD. Pro salute novi mundi: historia de la Organización Panamericana de la Salud. Washington DC, Oficina Sanitaria Panamericana, 1992. p. 296.

05. RODRIGUEZ, M. I. The International Health Program of the Pan American Health Organization. In: International Health: A North-South Debate. Washington DC, Panamerican Health Organization, 1992. p. 127-49.

06. SEAH, S. K. K. Canada and international health. Can.Med.Assoc.J., v. 115, p. 377-78,1976.

07. TERRIS, M. Epidemiology and leadership in public health in the Americas. J.Pub.Health Policy., v. 9, p. 250-60, 1988.

08. UNITED NATIONS DEVELOPMENT PROGRAM (UNDP). Human development report 1990. New York: Oxford University Press, 1990. 